\title{
Page Rank based Design and Implementation of Search Engine Optimization
}

\author{
${ }^{1}$ Karthikeyan, K \& ${ }^{2}$ Sangeetha, M \\ ${ }^{1} \mathrm{Head}$, Department of Information Technology \\ ${ }^{2}$ Research Scholar in Computer Science \\ Dr. SNS Rajalakshimi College of Arts \& Science \\ Coimbatore - 641 049. TamilNadu, India.
}

\begin{abstract}
Search Engine Optimization (SEO) is an Internet marketing strategy and a process, widely used now-a-days for improving the volume or quality of traffic to a website through search engines. The results generated by search engines can be natural (organic or algorithmic) and/or paid search. In the present study, different techniques were used for achieving better optimization for Web 2.0 based websites. Different techniques related to SEO like keyword discovery, crawling, on-page and off-page optimization and different Google tools are discussed in this paper. The present study also study also describes the impact of SEO on Internet marketing. The results of the investigation help webmasters to gain a deep insight about SEO and also to guide them in making better decisions regarding their online advertising and marketing campaign.
\end{abstract}

Keywords: search engine optimization, top rankings, search engine, keyword placement, website visibility

\section{INTRODUCTION}

Search engines such as Google are the main way for the Internet users to find web sites. In this search engine, it is crucial for any web site to appear in the search results in order to attract website visitors. Since search engine users are more likely to click on a result at the top of the result page, being present only is not the optimal situation. A high search engine ranking is important for attracting traffic from search engines. This paper focuses on the impact of a high search engine ranking on the amount of visitors from the search engine and what value comes with these visitors. Therefore the methods for increasing search engine traffic were reviewed in the first part of this study. Based on this information the theoretical framework is constructed and three propositions are drawn up. The second part of this study consists of a multiple case study in which these propositions were tested. During the test period the search engine ranking for the two test cases, the amount of visitors from search engines and the sales figures were measured on a weekly basis. The results were then used to validate the developed research propositions.

Search Engine Marketing (SEM) can be applied to increase search engine visibility of a web site. When the search engine visibility is increasing the chance that a web site is visited increases as well, hence search engine marketing increases web site traffic. There are two clearly distinctive types of search engine marketing. First is search engine optimization (SEO) which improves volume and quality of web site visitors through the natural search results, also called organic listings. The advantage of SEO is that the visitors from organic search are free of charge, however it can require a time consuming process to gain and preserve a top search engine ranking. The second type of
SEM is search engine advertising (SEA) which is based on actually paying for advertisements next to the organic search results. This method has the benefits of speed and flexibility as a campaign can be set up almost instantly.

In this study, SEO practices have contributed to an increase in search engine rankings for a test case. This resulted in a significant increase in traffic from the search engines. In order to make search engine marketing to be profitable, the additional traffic should also result in increasing value for the web site. It is widely proved that higher search engine ranking attract more targeted traffic, as these visitors are actively searching for a product or service. However it must also be noted that an increase in search engine ranking leads to an increase in less relevant traffic as well. This could be explained by the fact that more accidental or explorative visitors will find and visit the web site. This has the consequence that even though the amount of visitors from search engines rises, the individual visitor on average seems to be of a lesser value. This in turn dampens the increase of the total amount of added value.

As more and more Internet users draw on search engines to navigate over the World Wide Web, the importance of these search engines is still increasing. According to Nielsen Ratings, 81 percent of the global Internet population visited a search engine in December 2006 (Thurow,2007) and this figure is still increasing. When an Internet user makes use of a search engine, he or she enters a certain phrase into the search engine. This phrase is commonly called a site's keyword or keyword phrase. When a keyword is entered into a search engine the search engine determines the most relevant results for that query and display the results in order of supposed relevancy. Generally search engines displays ten results in a page, dividing numerous results over various result pages.

Eye tracking studies (Gisbergen et al.,2006) have shown that search engine users generally see the top results but omit to look any further. In this study the methods to increase search engine visibility and traffic were discussed. The effectiveness were discussed in two subsequent stages. First, the effects of search engine ranking on the amount of website visitors are construed. Second, the effects of an increase in the number of web site visitors on conversion are discussed.

In search engines, users can search the index by typing the keywords to specify what they are searching for. Any pages that are deemed relevant by the search engine's algorithm are then listed and by clicking on a hyperlink the user will be taken to the site. This means that a search engine is build up out of three basic components: a spider, an index and a search interface. The spider or robot is an automated script, which browses the Internet in a methodical, automated manner. If the spider finds any web page, it is stored in the index of the search engine, after 
that the spider follows all hyperlinks on the page to index the underlying web pages. The index of the search engine is the one where the collected data are stored. When a search engine user performs a search he is not searching the web, but the cache of the web in the search engine's index. The search interface displays the results in the order that is determined by the search engine's algorithm. This algorithm is a complex formula which determines the relevancy of a web page to the submitted search query based on numerous known and unknown aspects.

According to Thurow (2007) search engine optimization and search engine advertising are the most relevant search engine marketing activities. Search engine optimization deals with three main components of search engine algorithms which are the text component, the link component and the popularity component

\section{METHODOLOGY}

The goals of the study are two folded, first to understand how search engine ranking can be influenced in the most profitable manner, second find out the effect of an increasing search engine ranking. There are two methodologies in which literature review are executed and multi-case studies are carried out. There is some knowledge questions like

1. What are the different methods of influencing search engine ranking?

2. What is the effect of each method on search engine ranking?

3. How can the added value of a high search engine ranking for a web site be defined?

To answer these knowledge questions of the research problem- a literature review was conducted to cover the aspects of search engine ranking. In the review of literature both search engine ranking and search engine marketing were discussed.

There are some propositions which are part of the case study which will be performed to answer the main research question. According to Yin (2003) case studies are the preferred research strategy when a 'how' question is being posed, when the investigator has time control over the events, and when the focus in on a contemporary phenomenon within some real-life context. The case study is a way of investigating an empirical topic by following asset of prespecified procedures, these procedures put out in the following paragraphs.

As mentioned in the introduction of this methodology there is a distinction between the research method for the first research questions and the methods for the second part of this research. The first knowledge question is answered using an extensive explorative literature review on possible approaches to increase the amount of visitors to a web site through search engines. The starting point of this exploration has been the book 'Search Engine Visibility' by Thurow (2007), as this book discussed all basic methods to improve search engine positioning. The most potent methods of improving traffic towards a web site through search engines are discussed in the review of literature by means of their ability to improve search engine rankings. With this analysis the second knowledge question from the introduction are answered.

\subsection{Case Study Design}

In a case study five components are especially important during the design process. The first component is that, the study questions should be well formulated. This resulted in the main question capturing how a web site's search engine ranking influences the amount of visitors from search engines and the value of these visitors. The fact that the question is in a 'how' form also empowers the plan to answer it by means of a case study. Second component is that, propositions have been established at the end of methodology to answer the question and find the right direction for the research. Collected data are analyzed and judged against these propositions in order to answer the main question.

The propositions are as follows:

Pl: An increase in search engine ranking will result in a significant increase in the amount of visitors to a web site through the search engine.

P2: An increase in the search engine ranking of a web site will result in an increase in the added value per visitor.

P3: An increase in the number of visitors from search engines to a web site will result in an increase in the total added value of these visitors to the web site.

The third component consists the definition of what the 'case' is. As already discussed this study is based on a multi-case study. Within this study, the units of analysis are the web sites 'search engine rankings'. As each web site has a certain ranking in search engines for certain keywords it generates a certain amount of visitors which in turn contributes a certain value to the web site.

The case studies take place among customers of the affiliate marketing broker $\mathrm{M}^{4} \mathrm{~N}$ and are chosen based on availability. The two test cases are part of two different markets but both highly competitive markets. In this study the search engine ranking of the two test cases was optimized in two ways. The text component and the link component were analyzed on the web sites. On-site attributes were altered in such a way that the web sites itself were optimized for search engines. Then the link popularity of the two web sites mentioned above the research questions are answered based on findings in a case study. Because the stated theoretical framework does not focus on either critical or unique cases as well as does not imply to be representative for a wide base of cases, a singlecase design is not the optimal type of case study for this research.

The evidence from multiple cases were often considered for more convincing which then make the overall study to be regarded as more robust. With multiple cases it is also possible to follow replication logic, when uncovering a significant finding from a single case. This finding can be replicated in a second, third or even more cases. If each case gives similar results it strengthens the theoretical framework which is enhanced through a service of $\mathrm{M}^{4} \mathrm{~N}$. Within this service the affiliates are stimulated to refer to the web site of the advertiser in order to increase the popularity component. Therefore many web sites that are topically related or widely perceived as highly authoritative are contacted to ask for a link to the web site that is being promoted. The quantity of inbound link is not only important, but also the quality of these new links. Therefore the researcher closely monitored the quality and topical relevance of these newly created inbound links to ensure a beneficial effect on search engine ranking. The web sites that took part in this study are first been covered and after that the determination of the added value of additional visitors for that web site are discussed.

\subsubsection{Case \#1 - Sim Online}

The first web site is a sim online - telecommunication comparison site for consumers. Visitors can search for the cheapest offer available among many different web shops and 
will be forwarded to their selected offer. Sim Online is rewarded whenever a visitor from this web site actually performs a transaction on the web site. The number of these rewarded transactions measures the added value for this web site and this information is available through the back office of the organization. Data was collected over a period of 16 weeks in which a total of almost 10.000 visitors viewed over 46.000 pages.

\subsubsection{Case \#2 - Car Safety}

The second web site that is part of this research concerns an insurance company. Data was collected over a period of 25 weeks in which search engine ranking was enhanced from the tenth to the first place in Google.

\section{DATA ANALYSIS}

For each proposition the relevant data will be put together according to the procedures specified in the methodology. First the findings concerning search engine ranking and the number of visitors are first be analyzed to find patterns on proposition 1 . The search engine ranking and added value per visitor provides data for the second proposition and finally the total added value is the set off against the number of search engine visitors to test the third proposition.

\subsection{Collecting the Data}

According to the methodology, the data is collected from a variety of sources. For a period of 16 weeks the search engines ranking of the test case Sim Online have been measured using the software package Advanced Web Ranking. Simultaneously measuring the ranking the amount of visitors was drawn from both Google Analytics and Clicky. These figures were respectively placed in the column 'Visits Google Analytics' and Visits Clicky of this table. As data collection can be found, the figures in these two metrics can deviate, however it is important for the decision making process in this research that the trends in these two measures do correlate. Therefore the amount of visitors measured in Google Analytics and in Clicky was put together for both keywords and it were measured. The figure1 gives an indication of the correlation between the two measurements. Thereby the figures from Google Analytics are validated and it is safe to draw onclusions based on these numbers.

\subsubsection{Patterns in data on proposition $1(\mathrm{PI})$}

The first proposition states that an increase in search engine ranking results in a significant increase in the amount of visitors to a web site through the search engine. When a search engine ranking of a web site for a certain keyword is increasing this does not necessarily mean that this keyword rises through all possible rankings. For instance it is well possible that a web site's ranking for a certain keyword increases from the twentieth to the tenth place overnight. Also it must be noted that during this research search engine ranking was measured at an interval of one week. For these two reasons it is not possible to give an exact overview of increasing traffic as a result of improved search engine ranking. However as the research period was quite extensive, search engine traffic for certain keywords can be assessed on rankings for a more extensive period of time. In order to rule out coincidental and seasonal influences traffic was averaged over several weeks.

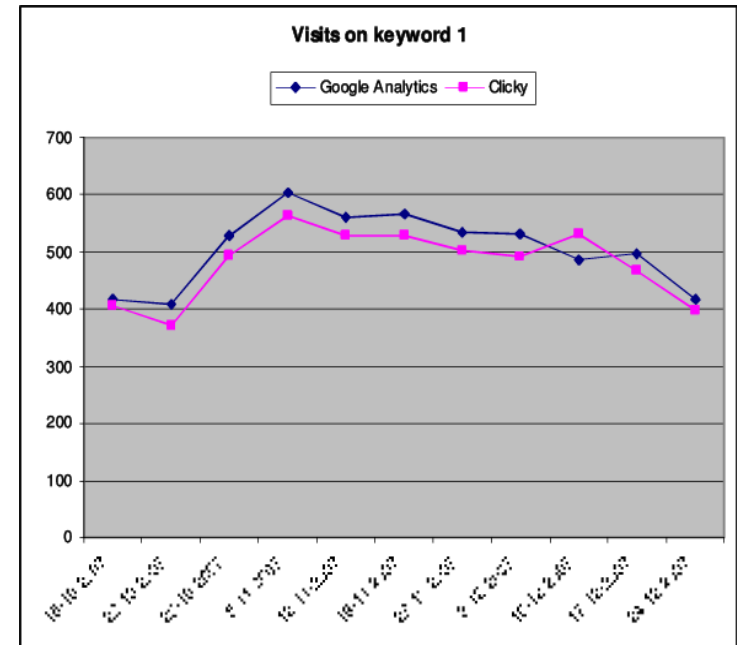

Figure 1-Analytical Chart

For the Sim Online case several keywords entered the top 10 in the search engine result pages of Google Netherlands. For each rank, the web site on the search engine result page and the amount of visitors from the search engine was measured. Each shift in search engine ranking towards the top results gives a significant increase of traffic. In order to test the stated proposition the results from the two test cases are compared. Therefore in both test cases the shifts in search engine rankings were combined with the relative amount of visitors from search engines. These relative amounts were calculated put into figure 2 below. The relative visitor amount $t$ was drawn from the AOL investigation.

In comparison to the AOL results the findings in the Sim Online case are slightly based towards the top results as a higher search engine rank results in more visitors than predicted based on the AOL case. It must be noted that Sim Online ranked on the fourth place in the search engine results at the end of the search engine optimization process, for this reason no results are available for the Sim Online case for the top three rankings. On the other hand there is the case of Car Safety in which did provide reliable results for the rankings all along the top five. In this last test case click through rates on the search result pages seem to be a bit more evenly spread. The bias towards the top results that was shown in the Sim Online case is even more clearly present in the case of Car Safety. Click through rates of the the second to the fifth place are far higher than in the AOL case.

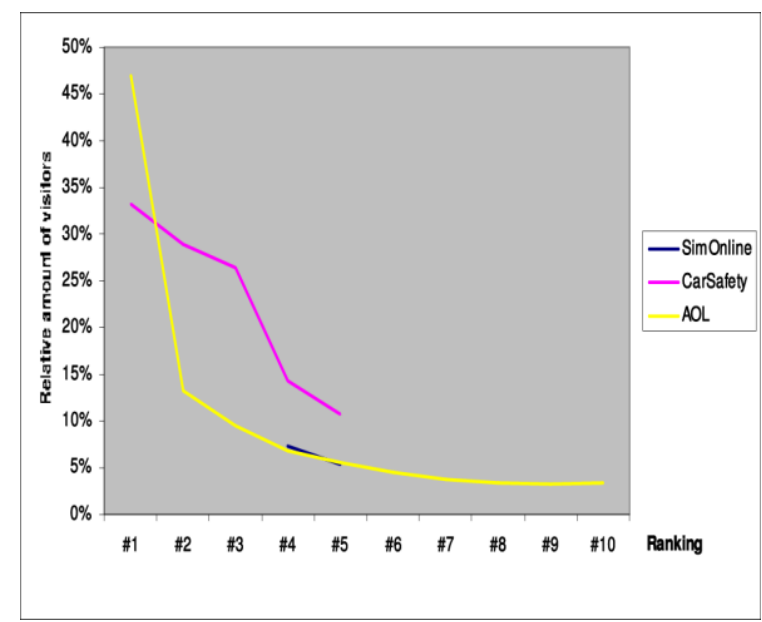

Figure 2 - The relative amount of visitors for each ranking in both test cases and the AOL results 
Based on the findings in the two test cases it is safe to say that an increase of search engine ranking results in over 5 percent additional visitors from search engines. For this reason the first proposition in this research is being accepted in these two cases affirming that an increase in search engine ranking will actually result in a significant increase in the amount of visitors to a web site through this search engine.

\begin{tabular}{|c|c|}
\hline Ranking & $\begin{array}{c}\text { Relative value per } \\
\text { visitor }\end{array}$ \\
\hline$\# 4$ & 79,94 \\
\hline$\# 5$ & 89,45 \\
\hline$\# 10$ & 100,00 \\
\hline
\end{tabular}

Table 1 - Relative amount of added value per value for the specific search engine rankings in case \#1 (Sim Online)

\subsubsection{Patterns in data on proposition $2(\mathrm{P} 2)$}

To get insight into the conversion rates of visitors who enter the web site through a search engine the second proposition was drawn up. It states that an increase in the search engine ranking of a web site will result in an increase in the added value per visitor. For this proposition the search engine ranking has been set out against the amount of added value per visit. Therefore the figures concerning ranking, visitor amounts and relative sales were given in the table 1.

The table depicted contains the analysis that was done for both keywords in the test case of Sim Online. The relative sales numbers were divided by the amount of visitors which resulted in the relative value per visitor for each specific search engine rank. Figures concerning sales or added value were not available in the test case of Car Safety, therefore the analysis and evidence for the second proposition is based on the Sim Online case only.

The results show that for both keywords in the Sim Online test case an increase in search engine ranking has resulted in a decrease of the added value per visitor. When the search engine ranking for keyword 1 increased from the 5th place to the 4th place in Google, the average sales per visitor went down from 7,68 to 6,86 . Comparable results were found when the ranking for keyword 2 increased from the 10th to the 5th spot: again the value per visitor dropped. Even though these findings seem to be based on just a few measurements, it must be stated that the figures in table 1 is aggregates of measurements of thousands of visitors and hundreds of sales over a period of 16 weeks. Based on these findings the proposition stating that an increase in search engine ranking will enhance the added value per visitor is rejected. In fact an increase in search engine ranking seems to result in a decrease of the value per visitor. As stated before an increase in search engine ranking implies getting more qualified traffic as search engine users are actively searching for a certain product or service.

However increasing search engine visibility also raises the issue that a less targeted audience will also find and visit a web site. This bulk traffic might even add value to the web site, however in general it seems to dampen the average value per visitor.

\subsubsection{Patterns in data on proposition $3(\mathrm{P3})$}

Next to determining whether increasing search engine ranking results in more web site visitors and what the effect of these higher rankings is on the average value of each visitor, it is also essential to verify whether an increasing search engine ranking will actually add value to the web site. Therefore the third proposition was put out to assess the relation between the number of visitors from search engines and the total added value of these visitors.

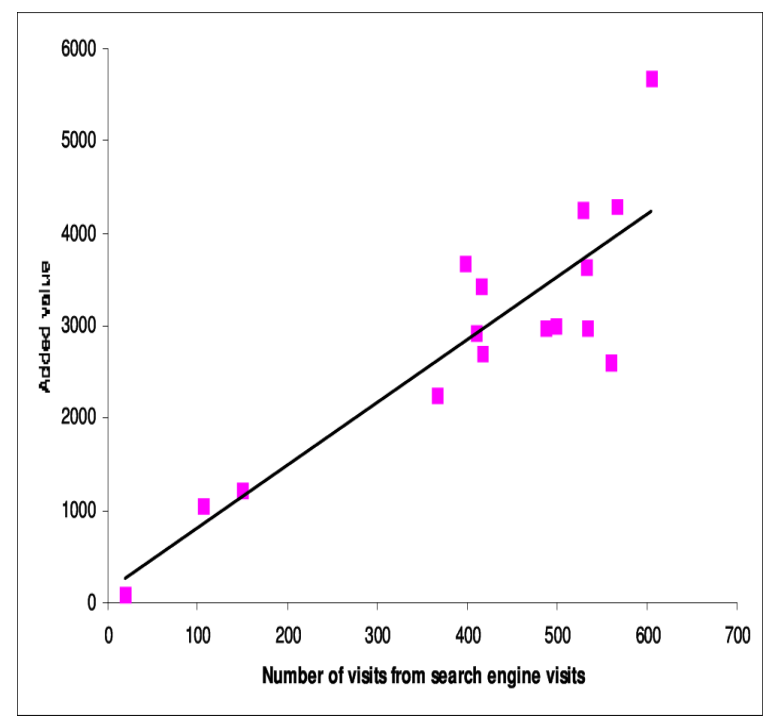

Figure 3 - Added value set of against the number of visits from search engine visitors

The third proposition states that an increase in the number of visitors from search engines to a web site will result in an increase in the total added value of these visitors to the web site. When the number of visits is put up against the total added value this results in figure 3 .

On the horizontal axis the number of visitors from search engines in a predefined period of time is set out. Although added value has not been increasing uninterrupted with the rising number of visits the trend line clearly shows that an increase in the number of visitors from the search engine has a positive effect on the total added value.

An extended version of table 2 is drawn up below showing that in the test case of Sim Online an increase in search engine ranking from the tenth to the fifth place resulted in over 63 percent additional visitors. The table 2 shows that the value of these visitors on average was decreased with 10.55 percent, resulting in an overall increase of almost 46 percent in total value when the keywords in this test case increase from the tenth to the fifth place on the search engine result page. 


\begin{tabular}{|c|c|c|c|}
\hline Ranking & $\begin{array}{l}\text { Relative } \\
\text { value per } \\
\text { visitor }\end{array}$ & $\begin{array}{l}\text { Relative } \\
\text { amount of } \\
\text { visitors }\end{array}$ & $\begin{array}{l}\text { Relative } \\
\text { total added } \\
\text { value }\end{array}$ \\
\hline$\# 4$ & 79,94 & 220,97 & 176,54 \\
\hline$\# 5$ & 89,45 & 163,08 & 145,79 \\
\hline$\# 10$ & 100,00 & 100,00 & 100,00 \\
\hline
\end{tabular}

Table 2 - Relative amount of total added value for the specific search engine rankings In case \#1 (Sim Online)

Even though the findings on the second proposition in this research instruct us that increasing traffic through higher search engine rankings dampened the average value per visitor. It is still safe to say that based on the findings in this research an increasing number of visitors from search engines will actually increase the total added value to a web site. Thereby the third and final proposition is accepted.

\section{SUMMARY}

In the data analysis, all three propositions were tested. In order to draw the conclusions based on reliable information first the figures of Google Analytics have been validated. Therefore the traffic amounts that were measured using Google Analytics were compared with those in Clicky. The results showed corresponding trends in both web analytic tools, which means that it is safe to draw conclusions based on those figures.

Based on findings in this research the first proposition was accepted, which means that an increase in search engine ranking actually results in additional web site traffic. Opposite to prior expectations the second proposition was rejected. A higher search engine ranking not only results in more relevant traffic but also attracted more bulk traffic which lowered the average value per visitor. Nevertheless did the increase of web site traffic outrun the decrease in value per visitor; therefore the overall value that was added to the web site by increasing its search engine rank grew significantly.

\section{CONCLUSION}

Search engine marketing can contribute remarkably to a web site's search engine visibility, which in turn can bring great value to a web site. The different methods of search engine marketing all have their own pros and cons. Search engine advertising has the benefits of speed and flexibility. An advertising campaign can be set up almost instantly and changes for a campaign are effective right away. However costs are associated with every single visitor that enters the web site through pay-per-click search engine advertising. On the other hand there is search engine optimization, which has the drawback being time consuming. It takes time to optimize a web site for search engines, acquire the required relevant back links and get these changes indexed by the search engine. Search engine optimization does not happen overnight, but opposite to search engine advertising in the end the visitors from organic search engine results are free. Within search engine optimization there are three main components. The text component and link component are part of the on-site process. These changes happen on the web site itself. The popularity component refers to the amount of external web sites that link to the web site that is being optimized. This aspect therefore is an off-site process. This popularity component is by far the most important aspect in search engine optimization.

By increasing search engine visibility the chance that a web site is visited is enhanced, hence SEM increases web site traffic. In order for search engine marketing to be profitable the increasing traffic should also result in increasing value for the web site. It is widely argued that higher search engine ranking attract more targeted traffic. The visitors from search engines are automatically more relevant visitors as they are actively searching for a product or service, however it must also be noted that an increase in search engine ranking may also lead to an increase in less relevant users. This could be explained by an increasing amount of accidental or explorative visitors among these additional visitors. This means that even though the amount of visitors coming from search engines rises, the individual visitors seem to be of a lesser value. This in turn dampens the increase of the total amount of added value. Nevertheless this research has shown that even though the decreasing value per visitor can dampen the growth of the total added value, the increase in web site traffic that is accompanied with increasing search engine rankings does overtake this is. Hence increasing search engine visibility has a positive effect on both the amount of visitors from search engines as well as the total value of these visitors.

\subsection{Implications for existing theory}

In this research three proposition based on existing theory were tested. The two theories stating that increasing search engine rankings result in more traffic and increasing added value were both confirmed to be correct. However theories which state that search engine visitors are automatically more relevant and more likely to convert into value for the website need to be reconsidered. Although increasing traffic from search engines does add incremental value to a web site, it must be noted that the average value per visitor decreases when search engine rank increases. A higher search engine ranking seems to result not only in more targeted traffic, but also happens to bring more bulk traffic along. Therefore any theory mentioning an increase in relevant traffic resulting from increasing rankings might as well incorporate that the average value per visitor decreases.

The results of this research are based on and limited to only two test cases in the Dutch market. This research is by no means intended to give a statistical analysis for the entire market of search engine marketing. In fact it would be interesting to see if similar results were to be found when more cases are to be added. Also it might be interesting to see whether similar analysis of added value can be made in international markets or for specific branches.

It also must be noticed that this research has focused on transactional value primarily. As there are also other methods for determining and measuring value it might be enlightening to see what impact search engine ranking has on value indicators like average time spending, bounce rates and the number of pages per visit. This might as well broaden the scope of this research towards informational web sites as well. With any luck this research has been an inspiration for other researchers to create a body of scientific knowledge in this area of online marketing.

The results of this study is based on and limited to only two test cases in the Dutch market. This study is by no means intended to give a statistical analysis for the entire market of search engine marketing. In fact it would be interesting to see if similar results 
were to be found when more cases are to be added. Also it might be interesting to see whether similar analysis of added value can be made in international markets or for specific branches.

It also must be noticed that this study has focused on transactional value primarily. As there are also other methods for determining and measuring value it might be enlightening to see what impact search engine ranking has on value indicators like average time spending, bounce rates and the number of pages per visit. This might as well broaden the scope of this research towards informational web sites as well. With any luck this research has been an inspiration for other researchers to create a body of scientific knowledge in this area of online marketing.

\section{REFERENCES}

[1] Brin, S., Page, L. (1998), The Anatomy of a Large-scale Hyper textual Search Engine, Computer Networks and ISDN Systems, Stanford.

[2] Chaffey, D., Ellis-Chadwick, F., Johnston, K., Mayer, R. (2008), Internet Marketing, Prentice Hall, London.

[3] Fain, D.C., Pedersen, J.O. (2005), Sponsored Search: a Brief History, Bulletin of the American Society for Information Science and Technology.

[4] Gisbergen, M.S. van, Most, J. van der, Aelen, P. (2008), Visual attention to Online Search Engine Results (available at http://www.checkit.nVonderzoek eyetracking.aspx).

[5] Granka, L., Joachims, T., Gay, G. (2004), Eye-Tracking Analysis of User Behavior in WWW Search, South Yorkshire.

[6] Hayward, N., Rogers, M. (2005), Search is brand, Web optimizer and Market Sentinel Ltd., London (available at http://www.weboptimiser.com/resources/Searchisbrand280 805.pdf).

[7] Jansen, B.J. (2005), Paid Search as an Information Seeking Paradigm, Bulletin of the American Society for Information Science and Technology.
[8] Jansen, B.J., Resnick, M. (2008), An examination of searcher's perceptions of non sponsored and sponsored links during ecommerce Web searching, Journal of the American Society for Information Science and Technology.

[9] Janssen, D., Heck, E. van (2007), How Will Online Affiliate Marketing Networks Impact Search Engine Rankings?, Erasmus Universiteit, Rotterdam.

[10] Laffey, D. (2007), Paid search: The innovation that changed the Web, Business Horizons.

[11] Lorigo, L., Pan, B., Hembrooke, H., Joachims, T., Granka, L., Gay, G. (2008), The influence of task and gender on search and evaluation behavior using Google, Information Processing and Management.

[12] Page, L., Brin, S., Motwani, R., Winograd, T. (1998), The PageRank Citation Ranking: Bringing Order to the Web, Computer Science Department, Stanford University.

[13] Pan, B., Hembrooke, H., Joachims, T., Lorigo, L., Gay, G., Granka, L. (2007), In Google we trust: Users' decisions on rank, position, and relevance, Journal of ComputerMediated Communication, 12(3), article 3

[14] Pandey, S., Roy, S., Olston, C., Cho, J., Chakrabarti, S. (2005), Shuffling a stacked deck: the case for partially randomized ranking of search engine results.

[15] Weinreich, H., Obendorf, H., Herder, E., Mayer, M., (2008), Off the beaten tracks: exploring three aspects of web navigation, ACM, New York

[16] Yin, R.K. (2003), Case Study Research: Design and Methods, Sage Publications, London.

[17] Wall,A.M(2007),Search Enginge Optimization Book

[18] Thurow, S. (2007) Search Engine Visibility, $2^{\text {nd }}$ edition, New Rifers, Barkeley.

[19] Animesh, A., Ramachandran, V and Viswanathan, S.(2007), An Empirical Investigation of the Performance of Online Sponsored Search Markets, ACM, New York. 